\title{
Extension of the IEC 61000-4-20 Annex C to the Use of Arbitrary Transient Signals
}

\author{
Niklas Briest, Student Member, IEEE, David Hamann, Member, IEEE, Heyno Garbe, Fellow, IEEE, \\ and Stefan Potthast
}

\begin{abstract}
Transverse electromagnetic (TEM) waveguides are predominantly used for emission and immunity tests. General requirements for TEM waveguides are given by the IEC 61000-4-20. Annex $C$ of the IEC 61000-4-20 specifies immunity tests based on high-altitude electromagnetic pulses with a double exponential waveform. This waveform's shape is sufficiently defined by the rise time and the pulsewidth. The quality of its transmission within a waveguide can, thus, be expressed by the allowed variation of these parameters. However, other arbitrary signals cannot be reduced to just these characteristic parameters. In this paper, a method is described that offers the possibility to characterize the transmission quality of a TEM cell for arbitrary transient waveforms. It is based on the Pearson correlation coefficient of a so-called reference signal and the signals being measured within the test volume of a TEM cell. Both signals are measured simultaneously with identical field probes in order to be independent from the reproducibility of the signal generator. The signals are windowed and limited to include only the defining reflections and distortions. By means of this signal-dedicated validation procedure, the transmission quality of a TEM waveguide can be validated for an arbitrary transient waveform
\end{abstract}

Index Terms-High-altitude electromagnetic pulses (HEMP), intentional electromagnetic interference (IEMI), signal dedicated verification ( $\mathrm{SiDeV}$ ), transverse electromagnetic (TEM) waveguide validation, transients.

\section{INTRODUCTION}

W ITH their high degree of interconnectivity, modern electronic systems are prone to intentional or unintentional electromagnetic interference (IEMI). Therefore, emission and immunity measurements of all parts of a system are essential

Manuscript received December 1, 2016; revised January 17, 2017; accepted February 6, 2017. Date of publication March 29, 2017; date of current version April 20, 2017. This work was supported by the Bundeswehr Research Institute for Protective Technologies, NBC-Protection in Munster, Germany, under Contract E/E590/DZ011/CF011. This paper is for the Special Issue and is an expanded version from the 2016 IEEE Symposia from Ottawa.

N. Briest is with the Institute of Electrical Engineering and Measurement Technology, Leibniz Universität Hannover, 30167 Hannover, Germany (e-mail: briest@geml.uni-hannover.de).

D. Hamann is with IAV GmbH, 38518 Gifhorn, Germany (e-mail: david.hamann@ieee.org).

H. Garbe is with the Institute of Electrical Engineering and Measurement Technology, Leibniz Universität Hannover, 30167 Hannover, Germany (e-mail: heyno.garbe@ieee.org).

S. Potthast is with the Bundeswehr Research Institute for Protective Technologies and NBC Protection (WIS), 29633 Münster, Germany (e-mail: stefanpotthast@ bundeswehr.org).

Color versions of one or more of the figures in this paper are available online at http://ieeexplore.iee.org.

Digital Object Identifier 10.1109/TEMC.2017.2666880

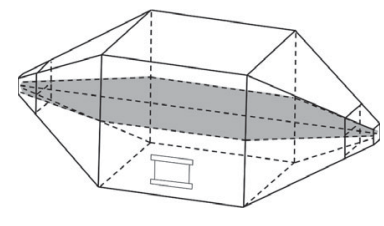

(a)

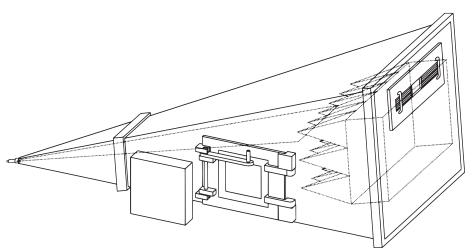

(b)
Fig. 1. Schematic of TEM waveguides. (a) Crawford cell. (b) GTEM cell [3].

in order to prevent system failures or even breakdowns. Some of these IEMI measurements, especially the high-altitude electromagnetic pulse (HEMP) immunity tests, are well described for the transverse electromagnetic (TEM) cells [1]. There are several designs of TEM waveguides [2]. Most widely used are the two-port waveguide, Crawford cell [see Fig. 1(a)], and the one-port waveguide gigahertz TEM (GTEM) cell [see Fig. 1(b)].

General requirements and a validation procedure for TEM waveguides are described in the IEC 61000-4-20 ed.2.0 [2]. That validation procedure is based on two methods and three figures of merit.

1) Method 1: The TEM mode-expressed by the ratio of the so-called secondary and primary field components-and the field uniformity - expressed by the standard deviation of the primary field component-are to be evaluated in a defined test section. The evaluation of these two figures is mandatory for all TEM waveguide measurements and is carried out in the frequency domain (FD).

2) Method 2: For the HEMP tests, the time-domain (TD) characteristics of the waveguide are expressed by means of the rise time $\left(t_{\text {rise }}\right)$ and the pulsewidth $\left(t_{\mathrm{fwhm}}\right)$ definitions for the electric field strength when a double exponential pulse with a defined waveform is applied to the port of the waveguide. Unfortunately, this third figure of merit describing the waveguide quality with respect to HEMP testing is strongly linked to the HEMP pulse.

For communication systems in particular, new waveforms have already evolved and an even larger multiplicity of waveforms is likely to be developed in near future. Not all of these waveforms can be sufficiently represented by the double exponential pulse and its wideband characteristics. For many signals, it is not even possible to define the parameters $t_{\text {rise }}$ and $t_{\mathrm{fwhm}}$ and to compare them to the limits given in [2] for the double exponential pulse. Hence, a TEM waveguide qualification method is required, which can be applied to any arbitrary waveform. 
In order to understand the restrictions of the methods currently described in the standard, Section II deals with the measurement setup and validation procedure according to Section II-A in the FD and Section II-B in the TD [2]. In order to overcome the beforementioned restrictions, the original measurement setup is subjected to a variation, described in Section III, where Section III-A explains the measurement setup in detail. Based on the measurements performed with that setup, Section III-B describes a method that allows us to express the TD transmission behavior of the specific combination of waveguide and signal in one figure of merit. Section III-C discusses a threshold determination not only to qualify the transmission characteristics of the waveguide, but also to quantify the transmission quality of the waveguide for arbitrary transient signals. Some comments on possible reference values for this figure of merit are given and illustrated by a subset of representative measurement results in Section IV. Here, Section IV-A discusses measurements performed within a Crawford cell and motivates the use of a GTEM cell for ultrawideband (UWB) pulses (see Section IV-B).

\section{IEC 61000-4-20 ED.2.0 VALIDATION}

The validation procedure described in the IEC 61000-420 ed.2.0 can be subdivided into the common validation procedure described in Section $\mathrm{V}$ of the standard and the more specific HEMP validation procedure in Annex C [2]. Whereas the common procedure, performed in the FD in order to verify the basic transmission quality, is mandatory for all interference and susceptibility tests carried out with the waveguide, the HEMP validation is carried out in the TD exclusively for HEMP measurements.

\section{A. Waveguide Validation in the FD}

According to Section 5 of IEC 61000-4-20 ed.2.0, the TEM mode and the field uniformity have to be evaluated in a specified uniform area in order to verify the existence of an electromagnetic field with sufficient TEM characteristics. This test is performed with continuous-wave signals. By definition, the ideal TEM field in the testing volume of the waveguide represents the far field of an antenna, and its $E$-field consists only of a component in the $y$-direction [4]. Due to its symmetry and geometry, the real field distribution within a TEM waveguide can only meet this demand at the center of the cross section just above the floor of the TEM cell. However, the standard requires the ratio of the secondary field components $\left(E_{x}\right.$ and $\left.E_{z}\right)$ to the primary field component $\left(E_{y}\right)$ to be smaller than $-6 \mathrm{~dB}$ in at least $75 \%$ of the testing points. As for the field uniformity, at least $75 \%$ of the primary field components measured in the testing points have to be in a band of $6 \mathrm{~dB}$. The latter requirement can be evaluated statistically, where a normal distribution of the primary field component is assumed by the standard [2], [5]. In this case, the sample standard deviation $\sigma_{E}$ of the primary $E$-field component has to be smaller than $2.61 \mathrm{~dB}$. Exceptions to these rules are accepted for up to $5 \%$ of the test frequencies. More details on these rules and exceptions can be found in the standard as well as earlier work from Hamann et al. [6].

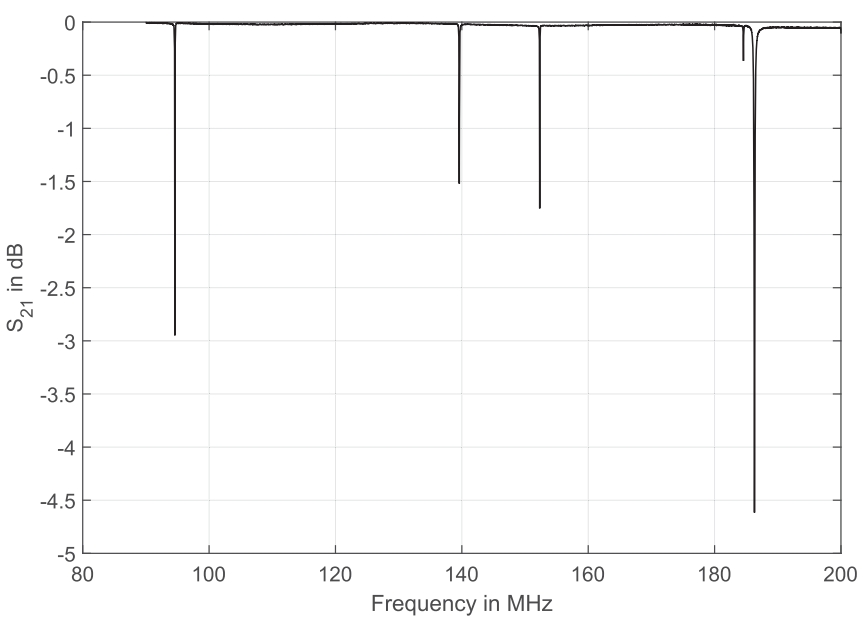

Fig. 2. $S_{21}$ of a Crawford cell [7].

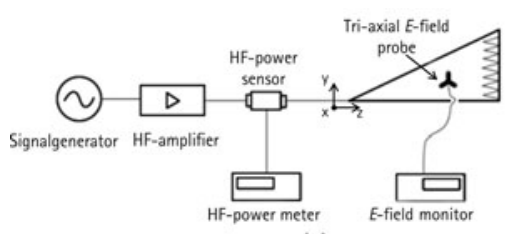

(a)

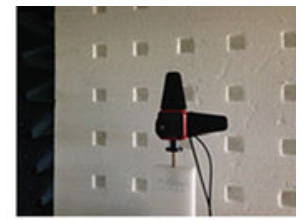

(b)
Fig. 3. Setup according to the IEC 61000-4-20. (a) Block diagram of the measurement setup. (b) Triaxial $E$-field probe in front of the uniform area.

1) Validation of a Crawford TEM Waveguide in the FD: To introduce the used Crawford cell [7], a measurement of the $S_{21}$ parameter is depicted in Fig. 2. The measurement is performed in a frequency range from 30 up to $500 \mathrm{MHz}$. The cut off frequency can be identified at a frequency of $94 \mathrm{MHz}$. Above $139 \mathrm{MHz}$, more resonances are coming up.

With respect to these results, the TEM field in this waveguide is expected to fulfill the validation criteria of IEC 61000-4-20 up to the cutoff frequency of $94 \mathrm{MHz}$. Above that frequency, the TEM mode can no longer be expected to be dominant.

Therefore, an investigation of the TEM mode within the Crawford cell is performed according to Section 5.2.2.3 "TEM mode verification" of the IEC 61000-4-20 ed.2.0 [2]. Therein, the three $E$-field components $\left(E_{x}, E_{y}\right.$, and $\left.E_{z}\right)$ are measured [see Fig. 3(b)] at nine positions with a triaxial $E$-field probe [see Fig. 3(a)].

The evaluation of the TEM mode is performed in the frequency range from $30 \mathrm{MHz}$ to $1 \mathrm{GHz}$, with a constant primary $E$-field $\left(E_{y}\right)$ strength of $10 \mathrm{~V} / \mathrm{m}$, and the ratio between the secondary and the primary $E$-field component is evaluated. The results are shown in Figs. 4 and 5 , by means of the ratio $\frac{E_{x}}{E_{y}}$ plotted and $\frac{E_{z}}{E_{y}}$, respectively. The TEM mode requirement is marked as a plane at $-6 \mathrm{~dB}$.

At the beforementioned cutoff frequency of $94 \mathrm{MHz}$, the ratios between the secondary $E$-fields $\left(E_{x}\right.$ and $\left.E_{z}\right)$ and the primary $E$-field $\left(E_{y}\right)$ do not exceed the limit of $-6 \mathrm{~dB}$ violated by the IEC 61000-4-20, even though a significant increase of the secondary components can be seen. The $-6 \mathrm{~dB}$ criteria 


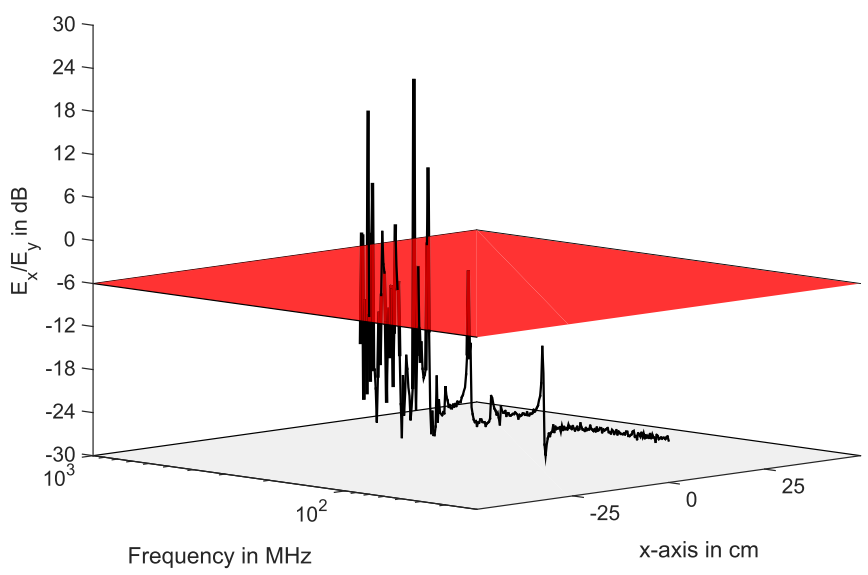

Fig. 4. $\frac{E_{z}}{E_{y}}$ ratio between secondary and primary $E$-field components in the Crawford cell at $y=200 \mathrm{~mm}$.

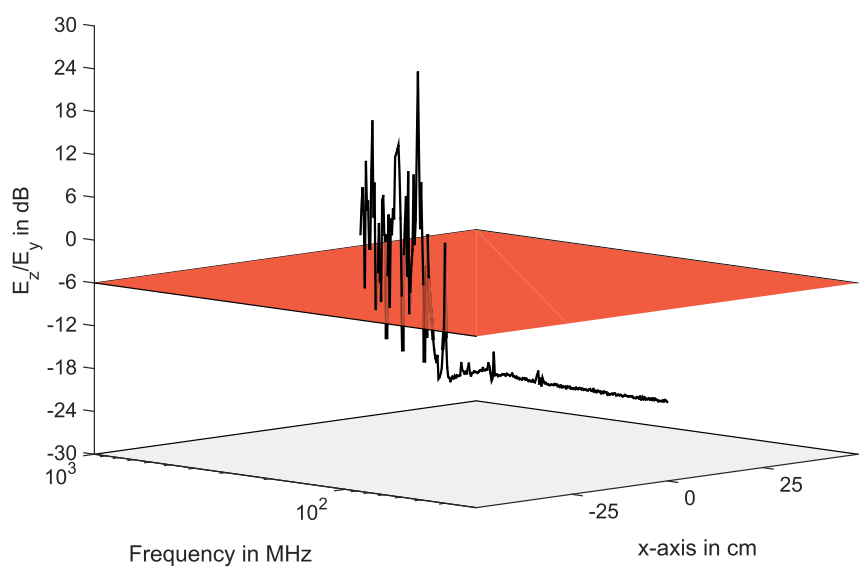

Fig. 5. $\frac{E_{x}}{E_{y}}$ ratio between secondary and primary $E$-field components in the Crawford cell at $y=200 \mathrm{~mm}$.

is not met at $187 \mathrm{MHz}$ with $\frac{E_{x}}{E_{y}} \approx-4,5 \mathrm{~dB}$ and at $230 \mathrm{MHz}$ with $\frac{E_{z}}{E_{y}} \approx-1,16 \mathrm{~dB}$. If the FD criteria are assumed to have an influence on the TD transmission characteristics, significant distortions are to be expected for transient signals covering frequencies above $187 \mathrm{MHz}$.

2) Validation of a GTEM1250 in the FD: In the next step, a closer look is taken at the results of the FD validation in a GTEM cell of the type GTEM1250 from Teseq. Hamann et al. [5] already investigated the GTEM1250 from Teseq in the FD and calculated the uncertainty contribution of the $E$-field homogeneity (see Fig. 6) - a value that can be understood as a best estimate for the standard deviation that is taken into account by the standard [2]. In general, the calculated uncertainty follows the requirement given with $\sigma_{\mathrm{E}} \leq 2,61 \mathrm{~dB}$. However, Fig. 6 shows significant peaks of the field homogeneity at specific frequencies (e.g., at frequencies around 100 and $400 \mathrm{MHz}$ ). It can be assumed that these TEM waveguide characteristics will have an impact on the transmission quality for arbitrary transient

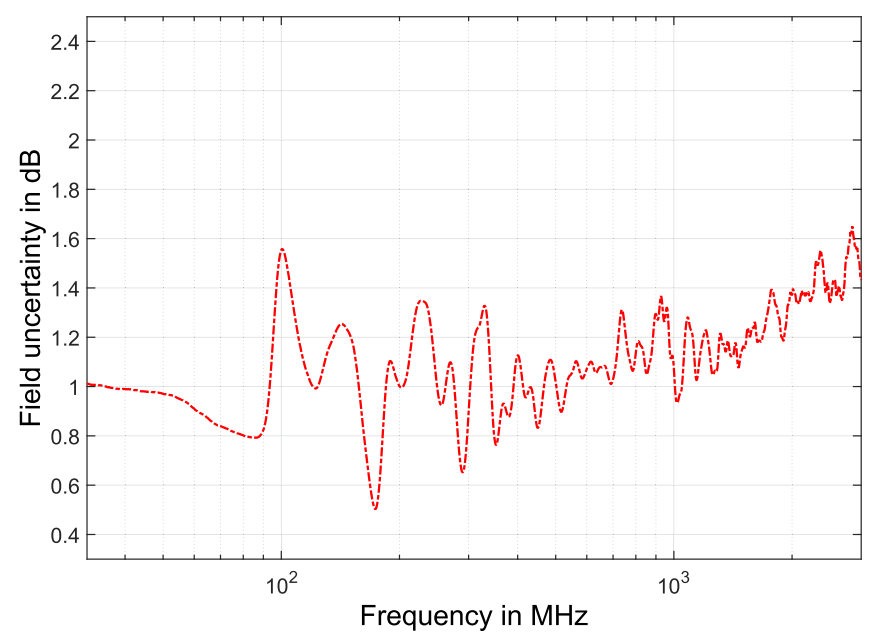

Fig. 6. Calculated field uncertainty for the GTEM1250 [8].

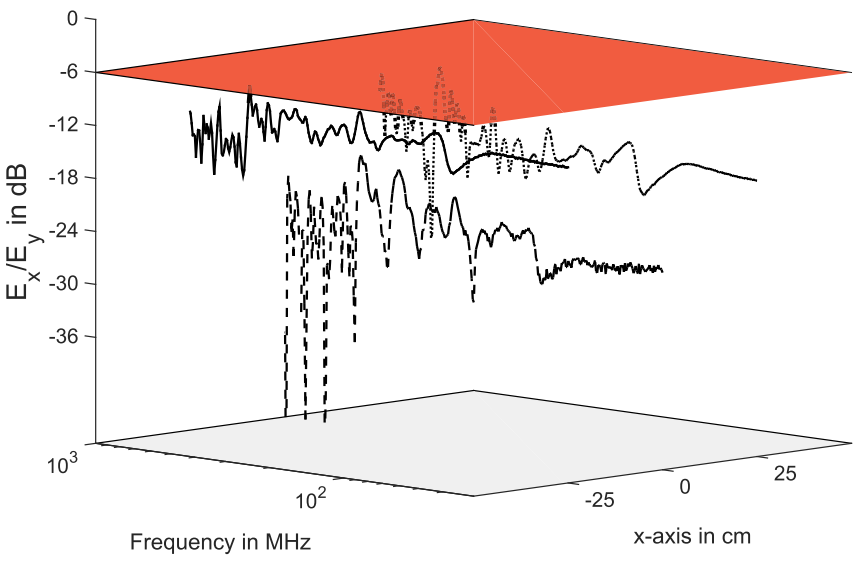

Fig. 7. $\frac{E_{x}}{E_{y}}$ ratio between secondary and primary $E$-field components at $y=400 \mathrm{~mm}[9]$.

signals, depending on their spectrum. It is necessary to evaluate the transmission characteristics of a TEM waveguide for each dedicated signal.

In the following, a discussion on basis of the TEM mode requirements according to the IEC 61000-4-20, as introduced in Section II-A1, is performed. Here, the uniform area is chosen at a septum height of $1 \mathrm{~m}$. Exemplary ratios $\frac{E_{x}}{E_{y}}$ (see Fig. 7) and $\frac{E_{z}}{E_{y}}$ (see Fig. 8) at three measuring positions with a height of $400 \mathrm{~mm}$ and $x=250 \mathrm{~mm}, x=0 \mathrm{~mm}$, and $x=-250 \mathrm{~mm}$ are depicted. The TEM mode requirement is marked by the plane at $-6 \mathrm{~dB}$.

The $E$-field ratio complies with the $-6 \mathrm{~dB}$ criteria for both secondary components at every measuring position and for all frequencies. However, equivalent to the uncertainty contribution of the field homogeneity, this ratio shows a large variation in the considered frequency range. The tabular data, which Fig. 8 is based on, show the maximum of the $E_{z}$-component at a frequency around $170 \mathrm{MHz}$. In relation to the investigations of field uncertainty, the secondary $E$-field components at the 


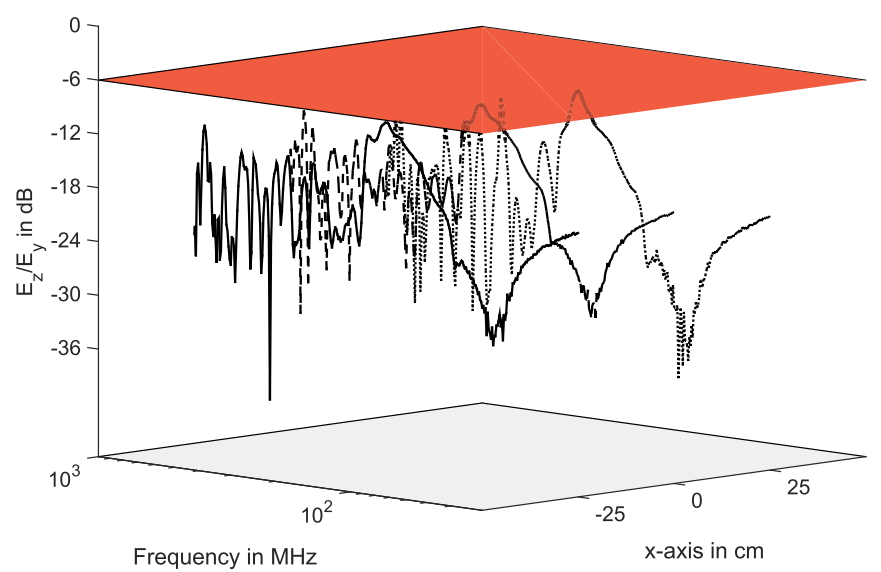

Fig. 8. $\frac{E_{z}}{E_{y}}$ ratio between secondary and primary $E$-field components at $y=400 \mathrm{~mm}[9]$.

distinctive frequencies (100 and $400 \mathrm{MHz}$ ) are examined, too. Especially, at $100 \mathrm{MHz}, E_{z}$ shows an increase that is strong in comparison to the other frequencies. In contrast, $E_{z}$ shows a rather small value at $400 \mathrm{MHz}$. Again, an impact of these variations on dedicated signals in the TD can be expected but hardly be quantified with FD measurements alone.

\section{B. Waveguide Validation in the TD}

Annex C of IEC 61000-4-20 provides requirements for transient signal testing in TEM waveguides. In this annex, a double exponential pulse with a rise time $\left(t_{\text {rise }}\right)$ and a pulsewidth $\left(t_{\mathrm{fwhm}}\right)$ is defined. The rise time accords with the time duration between $10 \%$ and $90 \%$ of the peak. The pulsewidth is defined as the time between the points where the leading, respectively, the falling edge reaches $50 \%$ of the waveform peak value. This waveform is given in (1) and reflects the waveform of a HEMP [2]

$$
E_{\mathrm{y}}(t)=E_{\mathrm{peak}} \cdot k\left(e^{-\beta t}-e^{-\alpha t}\right) \quad \text { in } \quad \frac{\mathrm{V}}{\mathrm{m}}[10] .
$$

According to this annex, the waveform of the primary $E$-field component $E_{\mathrm{y}}(t)$ measured within the test volume of the TEM waveguide has to be in accordance with the described waveform (1), with a maximum tolerance for $t_{\text {rise }}$ and $t_{\mathrm{fwhm}}$ [2]:

1) $t_{\text {rise }}$ : shall be $2.25 \mathrm{~ns} \pm 0.25 \mathrm{~ns}$;

2) $t_{\text {fwhm }}$ : shall be $27.5 \mathrm{~ns} \pm 2.5 \mathrm{~ns}$.

The tolerances for $t_{\text {rise }}$ and $t_{\mathrm{fwhm}}$ correspond to a certain level of distortion of the wideband characteristics of the pulse in the FD [11].

\section{Signal-Dedicated Verification (SiDeV) Procedure}

The verification method presented in this paper is signal dedicated and allows the evaluation of the quality of a distinct signal transmission in TEM waveguides in the TD. This section explains the recommended modifications to the IEC 61000-420 measurement setup, the mathematics that are based on the

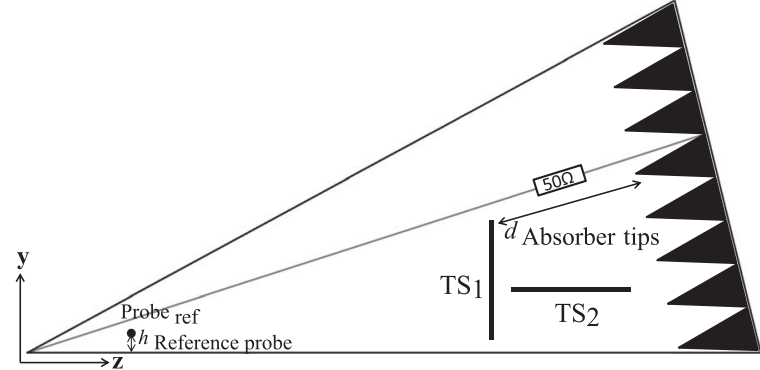

Fig. 9. Side view of the GTEM cell, with the two variants of test sections.

Pearson correlation coefficient (PCC). Some examples on possible outcomes of the validation procedure are given.

\section{A. SiDeV-Measurement Setup}

In order to evaluate the waveguide transmission quality, the reference signal and the signals in a test section have to be recorded. The test section is a part of the usable test volume of a TEM waveguide (see [2, Figs. D.7-D.11]) and depends on the uniform area as defined in the standard.

Two variations of test sections are discussed in this paper. The first possibility ( $\mathrm{TS}_{1}$ in Fig. 9) is equivalent to the uniform area as defined by the standard.

The number of measurement points for a transient TEM waveguide characterization has to be chosen according to Section 5.2.2 of the IEC 61000-4-20. At these measurement points, the primary $E$-field component $E_{y}$ shall be recorded in the TD.

The second variant of the test section $\left(\mathrm{TS}_{2}\right.$ in Fig. 9) gives a better insight into possible reflections from the absorbers at the back of the waveguide. For $\mathrm{TS}_{2}$, a separate coordinate system is included, $x^{\prime}$ and $z^{\prime}$. In detail, the measurement positions are placed at a height of $541 \mathrm{~mm}$ above the floor of the cell, which corresponds to the manufacturers recommendations [12]. $\mathrm{TS}_{2}$ has to be sampled in at least nine measuring positions.

The reference position is located as close to the feeding section as possible ( $z$-axis) and centered to the septum ( $x$-axis) (see Fig. 9). Along the $y$-axis, the reference position is located at the half of the septum height at the feeding section. In the frequency spectrum of interest, no higher order modes are able to propagate and no signal distortions will appear at the reference position. Thus, the reference signal accords to the signal supplied by the arbitrary signal generator.

The primary $E$-field $E_{y}$ is measured at the reference position and at the test section simultaneously. The performance of two identical field probes in both measurement positions is suggested to reduce the mathematical effort of correcting the measured field signals. They have to be small in comparison to the cross section of the waveguide in order to avoid field distortions, and their bandwidth shall cover that of the arbitrary transient waveform.

Both field probes are mounted on a positioning system, whose shaft dimensions are equal to the probes head [see Fig. 11(b)], what reduces the measurement uncertainty caused by the field probe position. The waveform of the reference signal $\left(E_{y, \mathrm{ref}}\right)$ 


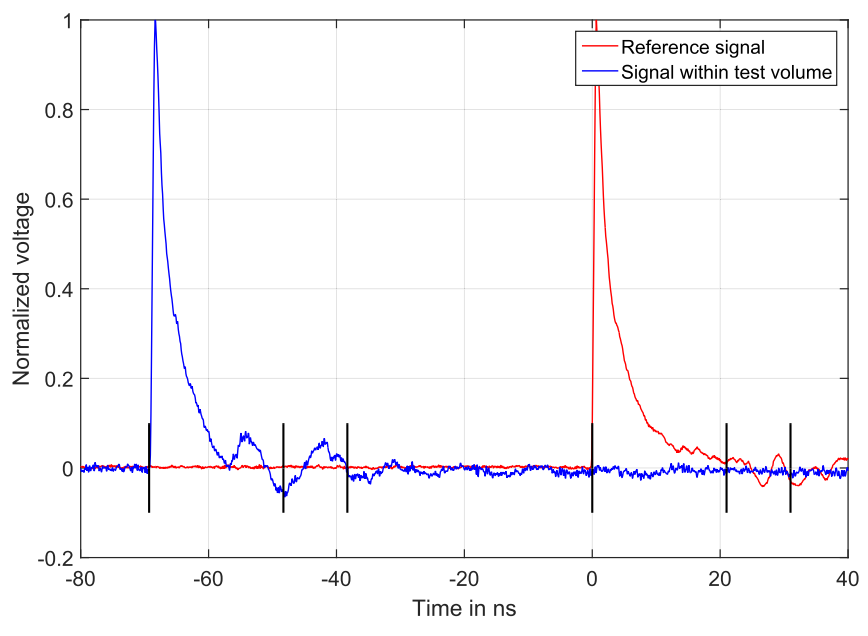

Fig. 10. Signal windowing according to (2) and (3).

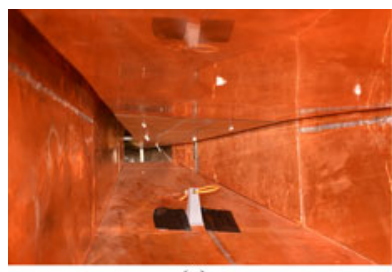

(a)

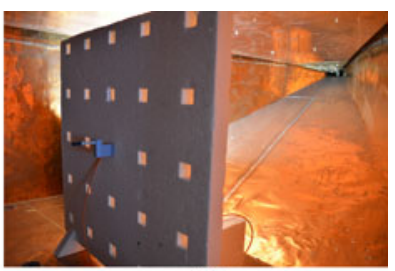

(b)
Fig. 11. Measuring positions within the Crawford cell according to $\mathrm{SiDeV}$ (see Section III-A). (a) Reference position. (b) Uniform area in the $x-y$ plane.

and the signal in the test section $\left(E_{y, \mathrm{ts}}\right)$ are recorded by a digital oscilloscope.

\section{B. SiDeV—Signal Postprocessing and Correlation}

In order to offer a quick evaluation based on just one qualifying parameter, every measured signal in the test section is correlated with the reference signal [13]. To do so, both signals have to be windowed. The goal of the windowing shall be that the remaining signal contains the most relevant information and-depending on the waveforms characteristics-reflections.

The windowing specifications given in the following passage can be understood as an example for transients with a double exponential envelope (see Fig. 10). The beginning $\left(t_{\text {rise }}\right)$ of the signal window for the reference signal and the signals within the test volume is defined by the minimum clearly detectable amplitudefor example, $5 \%$ of $E_{\mathrm{y}, \max }$. The signal length $\left(t_{\text {length }}\right)$ depends on the signal itself as well as on the location of the uniform area and its distance to the absorber tips. Therefore, the signal length is calculated for the signal within the test volume and is then applied to the reference signal to guarantee an equal signal length.

The time $t_{\text {length }}$ (see Fig. 9) consists of two parts:

$$
t_{\text {length }}=t_{\text {length }}+t_{\text {length }_{2}} .
$$

Here, $t_{\text {length }}$ represents the signal length of the voltage signaltime between the first rise exceeding, e.g., 5\% of the peak value $\left(t_{\text {start }}\right)$ till the last fall below $5 \%$. The second part $t_{\text {length }}$ is calculated as

$$
t_{\text {length }}=\frac{2 d_{\mathrm{abs}}}{c_{0}}
$$

where $d_{\mathrm{abs}}$ represents the distance to the absorber tips and $\mathrm{c}_{0}$ is the speed of light in free space. This value correlates to twice the propagation time from the measuring position to the absorber tips, ensuring that relevant reflections are covered by the signal window.

Once the signal is reduced to its most relevant part by the windowing, its samples $E_{y, \text { ref }}[k]$ and $E_{y, \text { ts }}[k]$ are normalized to their mean values $\bar{E}_{y, \text { ref }}[k]$ and $\bar{E}_{y, \text { ts }}[k]$ for the reference position and each point in the test section to its mean value:

$$
\begin{aligned}
E_{\mathrm{ref}} & =E_{y, \mathrm{ref}}[k]-\bar{E}_{y, \mathrm{ref}} \\
E_{\mathrm{ts}} & =E_{y, \mathrm{ts}}[k]-\bar{E}_{y, \mathrm{ts}} .
\end{aligned}
$$

In the next step, the windowed signals can be correlated on basis of (6):

$$
\rho\left(E_{y, \mathrm{ref}}, E_{y, \mathrm{ts}}\right)=\frac{\frac{1}{N} \sum_{k=1}^{N} E_{\mathrm{ref}} \cdot E_{\mathrm{ts}}}{\sqrt{\frac{1}{N} \sum_{k=1}^{N} E_{\mathrm{ref}}^{2}} \cdot \sqrt{\frac{1}{N} \sum_{k=1}^{N} E_{\mathrm{ts}}^{2}}} .
$$

This calculation can be performed for each test point, and the resulting values of the thus-obtained PCC can be visualized by means of a heat map covering the test section in order to obtain an overview of the transmission quality of the investigated waveguide. Such a heat map reveals the restrictions of the test volume and shows the influence of the waveguide's possible flows.

\section{SiDeV-Threshold Determination}

To quantify the level of distortion or to verify that a signal is transmitted shape inherent, a threshold has to be defined for the PCC. In the IEC 61000-4-20 Annex C, the double exponential pulse is defined by its rise time $t_{\text {rise }}$ and its pulsewidth $t_{\text {fwhm }}$, with the tolerances explained earlier.

An arbitrary signal generator is used to produce two double exponential pulses: one with $t_{\text {rise }}=2$ ns and $t_{\text {fwhm }}=$ $25 \mathrm{~ns}$ and the other with $t_{\text {rise }}=2.5 \mathrm{~ns}$ and $t_{\mathrm{fwhm}}=30 \mathrm{~ns}$. The values correspond to the two waveforms with the most diverse parameters still within the allowed tolerances. The waveforms are recorded by an oscilloscope and their PCC is $\rho=0.994$ ( $\hat{=} 99.4 \%$ ), which implies a deviation of $0.6 \%$ from a perfectly transmitted waveform.

Taking a typical measurement setup, consisting of an arbitrary transient signal generator, two field probes, and a digital oscilloscope, and the corresponding measurement uncertainty into account, the PCC should be no smaller than $\rho=0.9$, which can be understood as a reference value for any arbitrary transient test signal.

\section{EXAMPLES OF SiDEV PERFORMANCES}

The introduced validation method is performed for two types of TEM waveguides: the investigated Crawford (see Section IV-A) and GTEM cell (see Section IV-B). First, the 


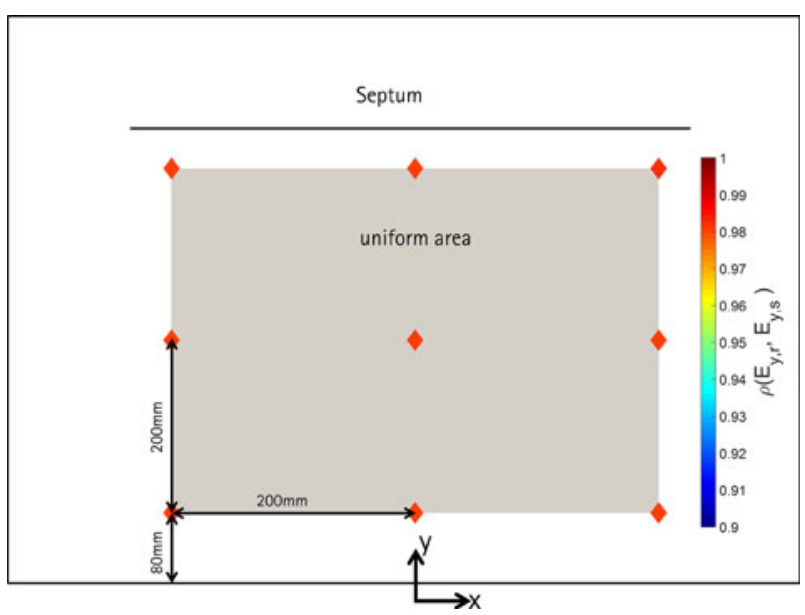

Fig. 12. PCC for a HEMP within the Crawford cell.

transmission quality of the Crawford cell for two different signals with a double exponential waveform is discussed. It is shown that measurements of UWB pulses have to be performed within GTEM cells. Furthermore, the GTEM1250 and its transmission quality for an UWB and a damped sinusoidal (DS) pulse are examined. By means of the DS, it is shown that the double exponential pulse according to the IEC 61000-4-20 Annex $\mathrm{C}$ cannot be used as a validating representation for any other transient signal.

\section{A. Crawford TEM Waveguide [7]}

Two measuring positions are arranged within the Crawford cell, as described in Section III-A. At the reference position [see Fig. 11(a)] and at each measuring position within the uniform area [see Fig. 11(b)], an optical $E$-field probe is placed. The $E$-field probes cover a frequency range of $500 \mathrm{kHz}-3 \mathrm{GHz}$.

The burst generator NSG 3040 from Teseq supplies a double exponential pulse, comparable to a HEMP, with the following specifications:

1) $t_{\text {rise }}: 5 \mathrm{~ns} \pm 20 \%$

2) $t_{\text {fwhm }}: 50 \mathrm{~ns} \pm 30 \mathrm{~ns}$.

According to the requirements and the introduced procedure of the $\mathrm{SiDeV}$ method, the resulting heatmap is depicted in Fig. 12.

In all nine measuring positions along the uniform area, a PCC of $\rho \approx 0.98$ can be calculated. This very high degree of shape consistency points out that this double exponential pulse can be used for tests in the Crawford cell. Fig. 13 shows the HEMP, measured at the reference position and within the test volume, in the TD.

The two signals diverge insignificantly from each other. Performing the $\mathrm{SiDeV}$ method, it is also possible to qualify a TEM waveguide for a signal, which has different defining parameters in comparison to the Annex C [2].

Furthermore, an UWB pulse, generated by a PBG3 from Kentech, is fed to the Crawford cell. The double exponential waveform is again defined by the rise time and the pulsewidth:

1) $t_{\text {rise }} \leq 100 \mathrm{ps}$;

2) $t_{\text {fwhm }} \approx 3 \mathrm{~ns}$.

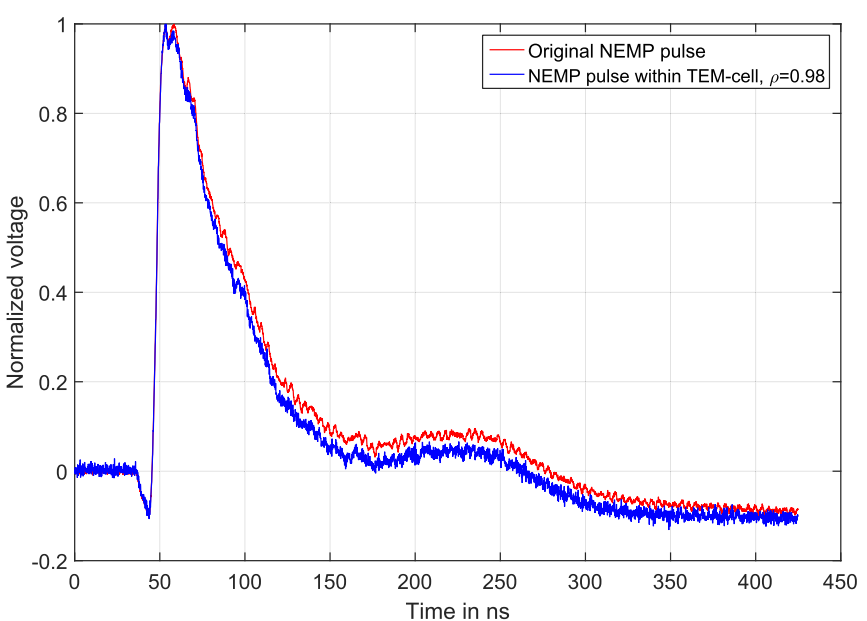

Fig. 13. HEMP at reference position and within the test volume of the Crawford cell.

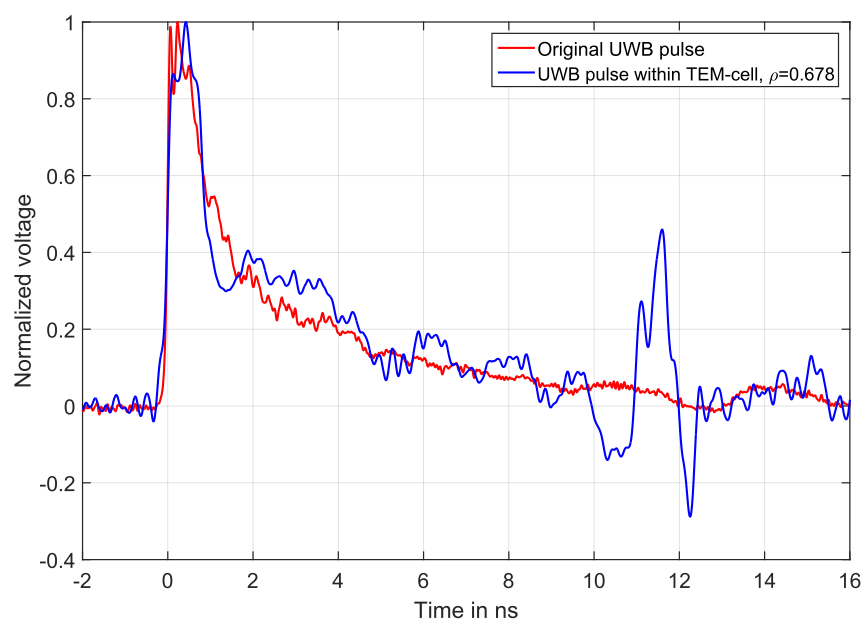

Fig. 14. Original UWB and within the test volume of the Crawford cell.

According to the investigation of the waveguide in the FD and taking the $t_{\text {rise }}$ and the $t_{\text {fwhm }}$ into account, a very low transmission quality is expected. Fig. 14 gives an idea of the distortions inflicted upon the UWB pulse.

In comparison to the original UWB pulse (see Fig. 14, red) measured at the reference position-the pulse measured within the test volume of the Crawford cell shows significant reflections after $10 \mathrm{~ns}$. These reflections can be identified by the $\mathrm{SiDeV}$ and result in a PCC of $\rho \approx 0.678$. With regard to the defined threshold (see Section III-C) for a sufficient transmission quality, the Crawford cell is obviously not suitable for an UWB transmission! Immunity measurements with such an UWB pulse have to be performed within a GTEM cell. The following section will discuss the use of the SiDeV method in such a GTEM cell and will verify if such an UWB pulse and its transmission in a TEM waveguide can represent the transmission for any other transient signal, included by the frequency spectrum of the UWB pulse. 


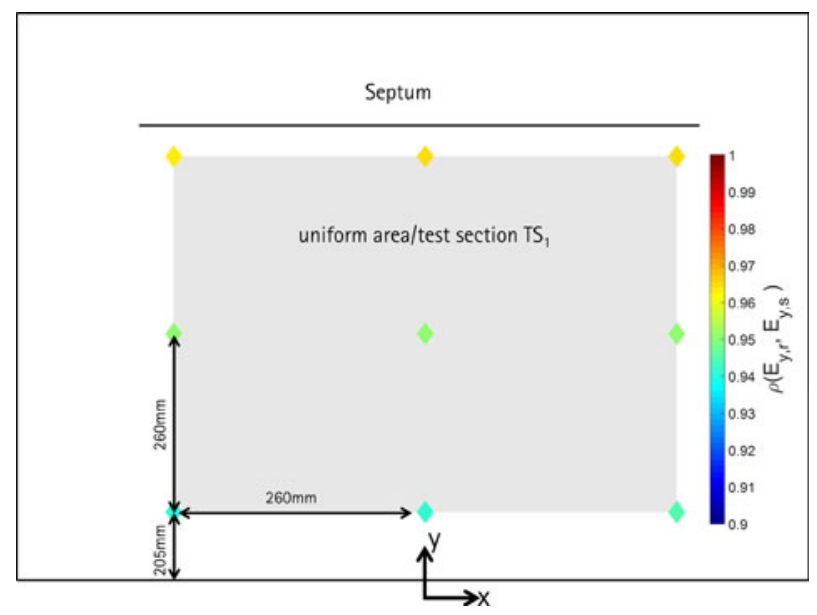

Fig. 15. PCC for a double exponential pulse at $\mathrm{TS}_{1}[9]$.

\section{B. GTEM Cell 1250 From Teseq}

In this section, $\mathrm{SiDeV}$ is applied to the combinations of two different waveforms with one GTEM cell, and the calculated PCCs are illustrated. In one instance, a double exponential pulse with a rise time $t_{\text {rise }} \leq 100 \mathrm{ps}$ and a pulsewidth $t_{\mathrm{fwhm}} \approx 3 \mathrm{~ns}$ is used. This pulse is measured with a D-Dot sensor. In the second measurement, a DS is generated by a vector signal generator. This waveform is measured with the optical $E$-field probe, already introduced in Section IV-A. In comparison to the ultrawideband double exponential pulse, the DS is a transient waveform with a rather narrow frequency spectrum, which is completely covered by the spectrum of the UWB pulse. Thus, it is possible to match the center frequency of the DS to critical frequencies of the considered TEM waveguide. Based on the previous investigations, the discussion of the field uncertainty [8], and the secondary $E$-field component $E_{z}$, two different center frequencies are chosen for the DS: The DS is generated with frequencies of 100 and $400 \mathrm{MHz}$, at which two different PCCs should be expected.

The previously introduced test sections $\mathrm{TS}_{1}$ and $\mathrm{TS}_{2}$ are considered. At $\mathrm{TS}_{1}$, the double exponential pulse is recorded with the D-Dot probes. According to the windowing specifications in Section III-B, the measured voltage samples $\dot{U}_{\text {ref }}\left(\sim E_{\text {ref }}\right)$ and $\dot{U}_{\mathrm{ts}}\left(\sim E_{\mathrm{ts}}\right)$ are edited and the PCC is calculated. Fig. 15 shows the PCC, including the measurement positions within the cross section of the TEM waveguide. The value of the PCC is represented by a color bar, where red corresponds to $\rho\left(E_{y, \text { ref }}, E_{y, \mathrm{ts}}\right)=1$ and blue to $\rho\left(E_{y, \mathrm{ref}}, E_{y, \mathrm{ts}}\right)=0.9$.

The PCC values for the double exponential pulse show an overall variation from $0.93(x=260 \mathrm{~mm}, y=205 \mathrm{~mm})$ to $0.97(x=-260 \mathrm{~mm}, y=725 \mathrm{~mm})$ in the test section $\mathrm{TS}_{1}$. Due to the symmetric orientation of the measurement positions in the test section $\mathrm{TS}_{1}$, the PCC should be the same for $x=$ $260 \mathrm{~mm}$ and $x=-260 \mathrm{~mm}$, along the $y$-axis for a homogeneous field distribution. Nonetheless, it can be seen that PCCs at $x=$ $260 \mathrm{~mm}$ are smaller than the PCCs at $x=-260 \mathrm{~mm}$. The reason for those smaller PCCs can be found in the position of the waveguide's door, which is placed on the right side of the cross section-where it is causing the lower PCCs.

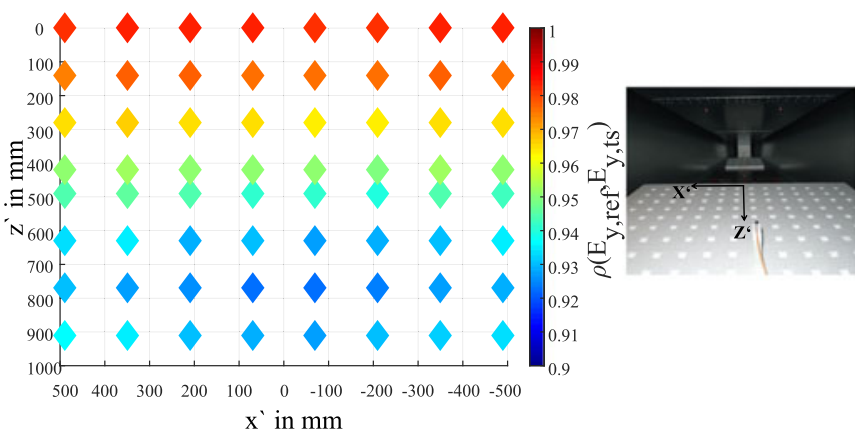

Fig. 16. PCC for a 100-MHz DS at $\mathrm{TS}_{2}$ [14].

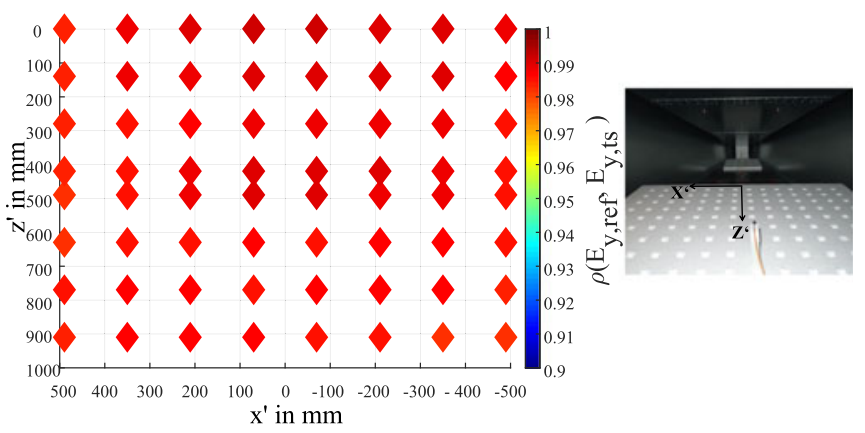

Fig. 17. PCC for a 400-MHz DS at $\mathrm{TS}_{2}[14]$.

At $\mathrm{TS}_{2}$, the DSs with center frequencies of 100 and $400 \mathrm{MHz}$ are evaluated. The DSs are measured with the optical $E$-field probes. For the PCC in the $\mathrm{TS}_{2}$, a symmetry along the $z^{\prime}$-axis (at $x^{\prime}=0 \mathrm{~mm}$ ) is expected, disregarding a lower PCC at the position of the waveguide door $\left(x^{\prime}=490 \mathrm{~mm}\right)$. Furthermore, an influence of the measured $E_{\mathrm{z}}$ component (see Fig. 8) on the PCC might appear. The PCC for the $100-\mathrm{MHz}$ DS is depicted in Fig. 16.

These values vary between $0.92\left(x^{\prime}=65 \mathrm{~mm}, z^{\prime}=780 \mathrm{~mm}\right)$ and $0.99\left(x^{\prime}=65 \mathrm{~mm}, z^{\prime}=0 \mathrm{~mm}\right)$ in the test section $\mathrm{TS}_{2}$.

Symmetric behavior of the PCC along the $z^{\prime}$-axis can be recognized, even though the influence of the waveguide's door again affects the magnitude of the PCC at $x^{\prime}=490 \mathrm{~mm}$, as it was presented for the $\mathrm{TS}_{1}$. Furthermore, a continuous decrease of the PCC along the $z^{\prime}$-axis can be recognized, which corresponds to distortions of the DS in TD [14]. The DS center frequency of $100 \mathrm{MHz}$, which was chosen with regard to the field uncertainty considerations [8] and to the discussion of the secondary Efield components in the FD, results in an imperfectly transmitted waveform shape.

In contrast to $100 \mathrm{MHz}$, the investigations for $400 \mathrm{MHz}$ in the FD show a comparable low contribution of the $E$-field uncertainty and low ratios of $\frac{E_{x}}{E_{y}} \approx-18,1 \mathrm{~dB}$ and $\frac{E_{z}}{E_{y}} \approx-23,9 \mathrm{~dB}$, averaged for $\mathrm{TS}_{2}$. Based on these facts, a higher PCC is expected. The calculated PCCs for the 400-MHz DS are depicted in Fig. 17.

For the $400-\mathrm{MHz}$ DS, a nearly constant PCC is calculated with maximum variations in the range of $0.98-1$. However, the influence of the waveguide's door at $x=-490 \mathrm{~mm}$ and a 
constant decrease of the PCC along the $z^{\prime}$-axis are identifiable. Nevertheless, higher PCCs are reached, which result in a nearly perfect transmitted 400-MHz DS in the TD [14].

\section{CONCLUSION}

TEM waveguides and their validation procedures are described by the IEC 61000-4-20. Edition 2 of this standard specifies the use of a TEM waveguide with FD as well as TD criteria. It is Annex $\mathrm{C}$ that includes the requirements for testing transient waveforms in TEM waveguides and their characterization and evaluation in the TD. Unfortunately, this procedure is restricted to a double exponential pulse with its significant parameters $t_{\text {rise }}$ and $t_{\text {fwhm }}$. However, not every transient waveform can be described by these two parameters. In this paper, FD investigations in a Crawford cell and a GTEM1250-two examples representing the most common types of TEM waveguides-are performed and the results are discussed. The Crawford cell has a well-defined cutoff frequency. The investigation of the GTEM cell identifies discrete frequencies with a comparably high contribution to the field uncertainty and higher secondary $E$-field components hinting the existence of higher order modes.

With regard to upcoming waveforms, a new qualification method that is applicable for any transient waveform is required. The so-called SiDeV-Signal Dedicated Validation procedure answers to this requirement and is presented in this paper. Therein, a two-antenna setup is used to measure the reference waveform, correlating to the supplied voltage signal and the waveform within the test volume. Postprocessing steps reduce the signals to their most relevant information, focusing on possible reflections and signal distortions. Based on these signals, the transmission quality of the TEM waveguide is evaluated by the calculation of the PCC. The PCC is displayed by means of a heatmap, which clearly reveals signal distortions.

Investigating a HEMP and an UWB pulse in a Crawford cell, it is shown that a defined cutoff frequency has a significant distorting effect to the UWB pulse, while the HEMP can be transmitted without any distortion. For immunity testing with UWB pulses, the performance of a GTEM cell is required.

The frequency investigations of the GTEM cell lead to the following statement: In the case of a well-transmitted wideband double exponential pulse, it cannot be assumed that any transient waveform, with a spectrum within the bandwidth of the double exponential pulse, will also be transmitted without deformation. The transmission of the double exponential pulse does not represent the transmission of arbitrary waveforms sufficiently.

This hypothesis is proved to be correct by feeding a narrowband signal-in this case, a DS with a center frequency of $100 \mathrm{MHz}$ - to the GTEM cell and evaluating the PCC in a test section. An increasing distortion of the $\mathrm{DS}$ - in the direction of the absorber tips - can be seen. Furthermore, the distorting effects of the waveguide door can be identified for both test sections introduced in this paper. However, the calculated PCC for the GTEM1250 lies within the defined threshold. This threshold is set on the basis of the PCC, calculated for two double exponential pulses, representing the worst-case distortions still allowed by the tolerances defined in Annex C of IEC 61000-4-20.
This paper clearly shows that the double exponential pulse and its wide frequency spectrum cannot completely specify the transmission quality of TEM waveguides for any transient waveform lying within the spectrum of the double exponential pulse. Therefore, it is recommended to perform the $\mathrm{SiDeV}$ method with the intended waveform shape.

This transient TEM waveguide transmission validation method was submitted to the Joint Task Force TEM waveguides and is proposed to be included as an informative annex supplementing the existing Annex C.

\section{REFERENCES}

[1] W. Radasky, K. Smith, D. Hansen, and D. Ristau, "Calculations and measurements of fast EM pulses in the GTEM cell," in Proc. IEEE Int. Conf. Electromagn. Compat., 1996, pp. 52-57.

[2] Testing and Measurement Techniques-Emission and Immunity Testing in Transverse Electromagnetric (TEM) Waveguides, IEC 61000-4-20 ed.2.0, 2010.

[3] J. Karst, C. Groh, and H. Garbe, "Calculable field generation using TEM cells applied to the calibration of a novel E-field probe," IEEE Trans. Electromagn. Compat., vol. 44, no. 1, pp. 59-71, Feb. 2002.

[4] C. A. Balanis, Antenna Theory: Analysis and Design, 3rd ed. New York, NY, USA: Wiley-Interscience, 2005.

[5] D. Hamann, M.-B. Konerding, and H. Garbe, "CISPR 16-4-2 equivalent measurement uncertainty analysis for TEM waveguides," IEEE Trans. Electromagn. Compat., vol. 57, no. 4, pp. 616-622, Aug. 2015.

[6] D. Hamann, H. Garbe, T. Pusch, and M. Suhrke, "A detailed study on TEM waveguides' field distribution and efficiency," in Proc. IEEE Int. Symp. Electromagn. Compat., 2015, pp. 881-886.

[7] M. Crawford and J. Workman, "Using a TEM cell for EMC measurements of electric equipment," NBS Tech. Note 1013, 2010.

[8] D. Hamann and H. Garbe, "Enhanced estimates of field distribution's uncertainty contribution for TEM waveguides," in Proc. IEEE Int. Symp. Electromagn. Compat., 2014, pp. 899-902.

[9] N. Briest, H. Garbe, and S. Potthast, "Extension of the IEC 61000-4-20 annex C to the use of arbitrary transient signals," in Proc. IEEE Int. Symp. Electromagn. Compat., 2016, pp. 829-834.

[10] "Description of HEMP Environment—Radiated Disturbance, IEC 610002-9 ed.1.0, 1996.

[11] D. Zamow, H. Thye, and H. Garbe, "Applicability of the IEC 61000-4-20 for transient testing with UWB signals," in Proc. Int. Symp. Electromagn. Compat., 2008, pp. 1-5.

[12] TESEQ, Definition of Measurement Points for Field Uniformity. [Online]. Available: http://www.teseq.de/produkte/GTEM-1250.php

[13] C. Koelling, D. Zamow, and H. Garbe, "A correlation method to extend the IEC 61000-4-20 for UWB measurements," in Proc. EMC Eur. York, 2011, pp. 159-163.

[14] N. Briest, S. Potthast, and H. Garbe, "Transmission characteristics of a TEM waveguide for transient signals by the use of a damped sinusoidal," Adv. Radio Sci., vol. 13, pp. 175-179, 2015.

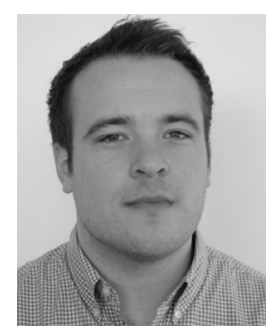

Niklas Briest (S'13) was born in Minden, Germany, in 1989. He received the Dipl.-Ing. degree in electrical engineering in 2014 from Leibniz Universität Hannover, Hannover, Germany, where he is currently working toward the Dr.-Ing. degree with the Institute of Electrical Engineering and Measurement Technology.

$\mathrm{He}$ is a member of the German National Committee on TEM waveguides and reverberation chambers and of the JWG TEM waveguides. His research interests include TEM waveguide measurements and microwave immunity measurements. 


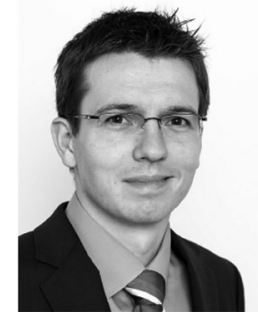

David Hamann (M'09) was born in Göttingen, Germany, in 1983. He received the Dipl.-Ing. and Dr.-Ing. degrees in electrical engineering from Leibniz Universität Hannover, Hannover, Germany, in 2009 and 2014, respectively.

From 2009 to 2014 , he did research on measurement methods and statistics of radiated emission test methods at Leibniz Universität Hannover. He is currently working in the research and development in the EMC \& Antenna Department at IAV GmbH, Gifhorn, Germany. In the executive committee of the IEEE German EMC Chapter, he is responsible for industry relations. He is a member of the German National Committee on TEM waveguides and reverberation chambers and Co-Convener of the JWG TEM.

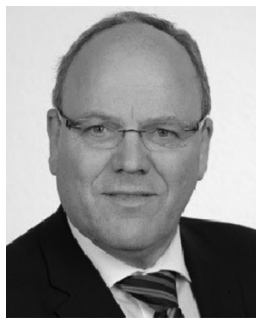

Heyno Garbe (M'89-SM'96-F'06) was born in Germany in 1955. He received the Dipl.-Ing. and Dr.-Ing. degrees in electrical engineering from the University of the Federal Armed Forces, Hamburg, Germany, in 1978 and 1986, respectively.

From 1986 to 1991, he was with the Asea Brown Boveri Research Center, Baden, Switzerland, where he was involved in several research activities on electromagnetic compatibility (EMC)-related topics. From 1991 to 1992, he was the Head of EMC Baden Ltd., Baden, Switzerland. Since 1992, he has been with Leibniz Universität Hannover, Hannover, Germany, where he is a Full Professor with the Department of Electrical Engineering and Informatics and Computer Science and holds the Chair of Electromagnetic Compatibility.

Prof. Garbe is very active in several EMC-related national and international standardization committees. He has been elected as EMP Fellow. He received the Laurence G. Cumming Award for Outstanding Service in 2003, the Richard R. Stoddard Award for Outstanding Performance in 2010, and the Honorary Life Membership in 2006 by the IEEE EMC Society. Since 1994, he has been a member of the Editorial Board of the IEEE TRANSACTIONS ON ELECTROMAGNETIC COMPATIBILITY. From 2010 to 2012, he served as the Editor-in-Chief of the IEEE TRANSACTIONS ON ELECTROMAGNETIC COMPATIBILITY. In 2015, he became the Vice-President of Communication Services for the IEEE EMC Society.

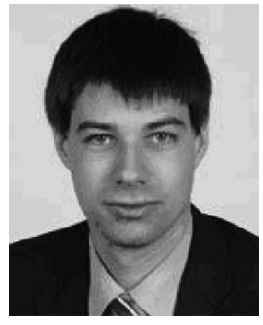

Stefan Potthast was born in Paderborn, Germany. He received the Dipl.-Phys. degree in physics, in 2003 and the Ph.D. degree in physics in 2007 from the University of Paderborn, Paderborn, Germany.

His research activities included epitaxial growth of cubic $\mathrm{GaN}$ and $\mathrm{AlGaN}$ and the design of a $\mathrm{Al}-$ GaN/GaN based HEMT. In 2007, he joined the Federal Office of Defense Technology and Procurement (BWB). Since 2008, he has been with the Bundeswehr Research Institute for Protective Technologies and NBC Protection (WIS) (in the Electromagnetic Effects Branch), Münster, Germany. His research interests include the influence of electromagnetic fields on electronic components and equipment. 